\title{
A STERILE NEUTRINO NEEDED FOR HEAVY-ELEMENT NUCLEOSYNTHESIS
}

\author{
DAVID O. CALDWELL \\ Physics Department, University of California, \\ Santa Barbara, CA 93106-9530, USA \\ E-mail: caldwell@slac.stanford.edu
}

\begin{abstract}
A neutrino mass-mixing scheme which successfully avoids the "alpha effect," allowing $r$-process nucleosynthesis in the neutrino-heated ejecta of supernovae, quite independently requires the same parameters as the scheme which best fits all current indications for neutrino mass. The significance for particle physics is this independent evidence for (1) at least one light sterile neutrino, $\nu_{s} ;(2)$ a near maximally-mixed $\nu_{\mu}-\nu_{\tau}$ doublet split from a lower mass $\nu_{\mu}-\nu_{s}$ doublet; (3) $\nu_{\mu}-\nu_{e}$ mixing $\gtrsim 10^{-4}$; and (4) a splitting between the doublets (measured by the $\nu_{\mu}-\nu_{e}$ mass difference) $\gtrsim 1 \mathrm{eV}^{2}$, favoring the upper part of the LSND range. If correct, it is tantalizing that neutrinos with tiny masses which mix with sterile species have profound effects on massive objects and the creation of the heaviest elements.
\end{abstract}

\section{Introduction}

While there is strong evidencel that the heaviest elements are produced in the neutrino-heated material ejected relatively long $(\sim 10 \mathrm{~s})$ after the explosion of a Type II or Type I b/c supernova, present calculations ditions which would prevent this rapid-neutron-capture (or $r$ ) process from occurring. Though general relativistic effectst and multi-dimensional hydrodynamic outflow have been invoked to solve these problems, these solutions are at best exceedingly finely tuned. In contrast, the solution 6 presented here is extremely robust, and the neutrino mass-mixing scheme it requires is exactly that needed if one is to explain all present evidence for neutrino mass.

In the next section, the particle physics motivations for that neutrino scheme are discussed, with special emphasis on recent results of the LSND experiment, since those are particularly important for the needs of the $r$ process. That is followed by a section on the $r$ process and its difficulties, after which the solution is presented.

\section{Particle Physics Evidence for a Four-Neutrino Scheme}

The need for at least one light sterile (i.e., not having the usual weak interaction) neutrino in addition to the known three active neutrinos was proposed as a way to explain the solar $\nu_{e}$ deficit, the anomalous $\nu_{\mu} / \nu_{e}$ ratio from at- 
mospheric neutrinos, and the apparent need for appreciable hot dark matter. The atmospheric anomaly, due to $\nu_{\mu} \rightarrow \nu_{\tau}$, requires a mass-squared difference between the $\nu_{\mu}$ and $\nu_{\tau}$ of $\Delta m_{\mu \tau}^{2}=0.003 \mathrm{eV}^{2}$ and maximal mixing $\left(\sin ^{2} 2 \theta_{\mu \tau}=1.0\right)$, with the latter property being quite important to solving the $r$-process problem, as shown below. The solar $\nu_{e}$ deficit is explained by $\nu_{e} \rightarrow \nu_{s}$ with $\Delta m_{e s}^{2} \approx 10^{-5} \mathrm{eV}^{2}$ and a small mixing angle $\left(\sin ^{2} 2 \theta_{e s} \approx 0.01\right)$ or $\Delta m_{e s}^{2} \sim 10^{-10} \mathrm{eV}^{2}$ and $\sin ^{2} 2 \theta_{e s} \sim 1$. The $\nu_{e}-\nu_{s}$ pair is of lower mass than the $\nu_{\mu}-\nu_{\tau}$ pair, which provide the hot dark matter. For the originally favored $\$$ critical-mass-density universe the $\nu_{\mu}$ and $\nu_{\tau}$ needed masses of around $2 \mathrm{eV}$ each.

This phenomenology was subsequently given theoretical bases in two 1993 papers 1 and has been utilized since in a large number of publications. Since that time the scheme has received support from the LSND experiment, the results of which provide some measure of the mass difference between the $\nu_{e}-\nu_{s}$ and $\nu_{\mu}-\nu_{\tau}$ pairs and hence of the neutrino contribution to dark matter.

In its 1996 publication, 10 LSND claimed a signal in $\bar{\nu}_{\mu} \rightarrow \bar{\nu}_{e}$ on the basis that 22 events of the type $\bar{\nu}_{e} p \rightarrow e^{+} n$ were seen, using a stringent criterion to reduce accidental coincidences between $e^{-}$or $e^{+}$and $\gamma$ rays mimicking the $2.2-\mathrm{MeV} \gamma$ from $n p \rightarrow d \gamma$, whereas only $4.6 \pm 0.06$ events were expected. The probability of this being a fluctuation is $4 \times 10^{-8}$. Note especially that these data were restricted to the energy range 36 to $60 \mathrm{MeV}$ to stay below the $\bar{\nu}_{\mu}$ endpoint and to stay above the region where backgrounds are high due to the $\nu_{e}{ }^{12} \mathrm{C} \rightarrow e^{-} X$ reaction. In plotting $\Delta m^{2}$ vs. $\sin ^{2} 2 \theta$, however, events down to $20 \mathrm{MeV}$ were used to increase the range of $E / L$, the ratio of the neutrino's energy to its distance from the target to detection. This plot was intended to show the favored regions of $\Delta m^{2}$, and all information about each event was used. The likelihood analysis applied did not have a Gaussian likelihood distribution, since its integral is infinite, but the likelihood contour labeled "90\%" was obtained by going down a factor of 10 from the maximum, as in the Gaussian case. The contours in the LSND plot have been widely misinterpreted as confidence levels - which they certainly are not-because they were plotted along with confidence-level limits from other experiments.

Recently the difficult, computer-intensive analysis in terms of real confidence levels has been done. 11 The likelihood for a grid in $\left(\sin ^{2} 2 \theta, \Delta m^{2}\right)$ space, including backgrounds, has been computed and compared with numerous Monte Carlo experiments to obtain a $90 \%$ confidence region. While the equivalency varies from point to point in the $\Delta m^{2}-\sin ^{2} 2 \theta$ plane, a typical value for the $90 \%$ confidence level is down a factor of 20 from the likelihood maximum. Thus the LSND allowed regions are considerably broader in $\sin ^{2} 2 \theta$ than in the plots published so far, and other experiments constrain allowed

pascos99: submitted to World Scientific on October 30, 2018 
$\Delta m^{2}$ regions less.

The confusion of comparing likelihood levels for LSND with confidence levels from other experiments may be exacerbated by using the $20-36 \mathrm{MeV}$ region for the LSND data. While this higher background energy range makes some difference for the 1993-5 data, it could have had an appreciable effect for the parasitic 1996-8 runs, which were at a low event rate, increasing the effect of cosmic ray background. This could raise the low end of the supposed signal energy spectrum, especially as the one LSND distribution which was statistically worrisome was the ratio $(R)$ of real to accidental events. Some accidental events in this $20-36 \mathrm{MeV}$ region would favor low values of $\Delta m_{e \mu}^{2}$ making the higher $\Delta m^{2}$ values desirable for dark matter appear less likely.

Nevertheless, when a joint analysis1 11 is made of the LSND and KARMEN 12 experiments even using the $20-36 \mathrm{MeV}$ range for LSND, the region around $5.5 \mathrm{eV}^{2}$ is as probable as the banana-shaped region at lower $\Delta m^{2}$, as shown in Fig. 1. Frequently ignored by theorists, this higher mass region is favored by the $\nu_{\mu} \rightarrow \nu_{e}$ LSND data. Of course in the $\nu_{\mu} \rightarrow \nu_{e}$ case 10 using $\nu_{\mu}$ from $\pi^{+}$decay in flight and detecting $\nu_{e}$ by $\nu_{e}{ }^{12} \mathrm{C} \rightarrow e^{-} X$, the backgrounds are higher and hence yield much poorer statistics than for $\bar{\nu}_{\mu} \rightarrow \bar{\nu}_{e}$ with $\bar{\nu}_{\mu}$ from $\mu^{+}$at rest. In addition to the $\Delta m^{2}$ issue, the important point of Fig. 1 is that although the KARMEN data are consistent with background, the joint analysis of the $\bar{\nu}_{\mu} \rightarrow \bar{\nu}_{e}$ data from the two experiments shows an appreciable region for a signal. KARMEN is continuing to take data, and LSND will have an improved analysis available soon. This new analysis has produced an excellent $R$ distribution for all the data and an energy distribution with reduced contributions at the low end, favoring higher $\Delta m_{e \mu}^{2}$ values than does Fig. 1 .

\section{Problems with Synthesis of the Heaviest Elements}

While in the next section we will find that the $r$ process of rapid neutron capture in supernovae provides strong support for the double doublet of neutrinos, initially the reverse appeared to be true, with the $r$ process apparently placing stringent limits on $\nu_{\mu}-\nu_{e}$ mixing. The origin of these limits is that energetic $\nu_{\mu}(\langle E\rangle \approx 25 \mathrm{MeV})$ coming from deep in the supernova core could convert via an MSW transition to $\nu_{e}$ inside the region of the $r$-process, producing $\nu_{e}$ of much higher energy than the thermal $\nu_{e}(\langle E\rangle \approx 11 \mathrm{MeV})$. The latter, because of their charged-current interactions, emerge from farther out in the supernova where it is cooler. Since the cross section for $\nu_{e} n \rightarrow e^{-} p$ rises as the square of the energy, these converted energetic $\nu_{e}$ would deplete neutrons, stopping the $r$-process. Calculations 13 of this effect limit $\sin ^{2} 2 \theta$ for

pascos99: submitted to World Scientific on October 30, 2018 


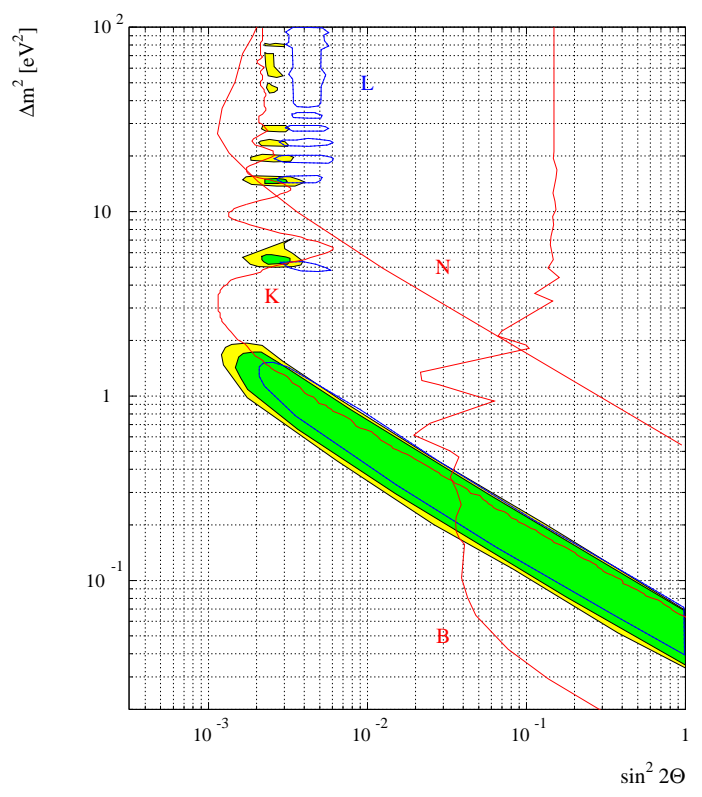

Figure 1. Filled in areas are $90 \%$ and $95 \%$ confidence regions based on the product of the KARMEN and LSND Feldman-Cousins likelihood ratios. Also shown is the FeldmanCousins $90 \%$ confidence region for LSND alone ("L"). Left of the "K", "N", and "B" curves are exclusion regions of KARMEN, NOMAD, and Bugey.

$\nu_{\mu} \rightarrow \nu_{e}$ to $\lesssim 10^{-4}$ for $\Delta m_{e \mu}^{2} \gtrsim 2 \mathrm{eV}^{2}$, in conflict with at least the higher mass region of the LSND results, which will be of particular interest here.

More recently, serious problems have been found with the $r$ process itself. First, simulations have revealed the $r$-process region to be insufficiently neutron-rich, since about $10^{2}$ neutrons is required for each seed nucleus, such as iron. This was bad enough, but the recent realization of the full effect of $\alpha$-particle formation has created a disaster for the $r$ process. region inside where the $r$ process should occur, all available protons swallow up neutrons to form the very stable $\alpha$ particles, following which $\nu_{e} n \rightarrow e^{-} p$ reactions reduce the neutrons further and create more protons which make more $\alpha$ particles, and so on. The depletion of neutrons rapidly shuts off the $r$ process, and essentially no nuclei above $A=95$ are produced.

To solve this problem the $\nu_{e}$ flux has to be removed before the $r$ process site, while leaving a very large $\nu_{e}$ flux at a smaller radius for material heating 
and ejection. The obvious difficulty of accomplishing this has led to searches for other possible sites for the $r$ process, such as neutron star mergers.

\section{Neutrino Solution for a Successful $r$ Process}

The apparent miracle of having a huge $\nu_{e}$ flux disappear before it reaches the radius of the supernova where $\alpha$ particles form can be accomplished $\theta$ if there is (1) a sterile neutrino, (2) approximately maximal $\nu_{\mu} \rightarrow \nu_{\tau}$ mixing, (3) $\nu_{\mu} \rightarrow \nu_{e}$ mixing $\gtrsim 10^{-4}$, and (4) an appreciable $\left(\gtrsim 1 \mathrm{eV}^{2}\right)$ mass-squared difference between $\nu_{s}$ and the $\nu_{\mu}-\nu_{\tau}$. This is precisely the neutrino mass pattern required to explain the solar and atmospheric anomalies and the LSND result, plus providing some hot dark matter!

Such a mass-mixing pattern creates two level crossings. The inner one, which is outside the neutrinosphere (beyond which neutrinos can readily escape) is near where the $\nu_{\mu, \tau}$ potential $\propto\left(n_{\nu_{e}}-n_{n} / 2\right)$ goes to zero. Here $n_{\nu_{e}}$ and $n_{n}$ are the numbers of $\nu_{e}$ and neutrons, respectively. The $\nu_{\mu, \tau} \rightarrow \nu_{s}$ transition which occurs depletes the dangerous high-energy $\nu_{\mu, \tau}$ population. Outside of this level crossing, another occurs where the density is appropriate for a matter-enhanced MSW transition corresponding to whatever $\Delta m_{e \mu}^{2}$ LSND is observing. Because of the $\nu_{\mu, \tau}$ reduction at the first level crossing, the dominant process in the MSW region reverses from the deleterious $\nu_{\mu, \tau} \rightarrow \nu_{e}$, becoming $\nu_{e} \rightarrow \nu_{\mu, \tau}$ and dropping the $\nu_{e}$ flux. For an appropriate value of $\Delta m_{e \mu}^{2}$, the two level crossings are separate but sufficiently close so that the transitions are coherent. Then with adiabatic transitions (as calculations show) and maximal $\nu_{\mu}-\nu_{\tau}$ mixing, the neutrino flux emerging from the second level crossing is $1 / 4 \nu_{\mu}, 1 / 4 \nu_{\tau}$, and $1 / 2 \nu_{s}$, and no $\nu_{e}$.

A more exact way to explain this in the four-neutrino formalism is to transform the four mass eigenstates into the flavor states via the mixing angles $\phi$ for solar $\nu_{e} \rightarrow \nu_{s}, \omega$ for LSND $\nu_{\mu} \rightarrow \nu_{e}$, and $\pi / 4$ (maximal mixing) for atmospheric $\nu_{\mu} \rightarrow \nu_{\tau}$. Symbolically,

$$
\left(\begin{array}{l}
\left|\nu_{s}\right\rangle \\
\left|\nu_{e}\right\rangle \\
\left|\nu_{\mu}\right\rangle \\
\left|\nu_{\tau}\right\rangle
\end{array}\right)=\left(\begin{array}{c}
\text { Atmospheric } \\
\nu_{\mu} \rightarrow \nu_{\tau}
\end{array}\right)\left(\begin{array}{c}
\text { Solar } \\
\nu_{e} \rightarrow \nu_{s}
\end{array}\right)\left(\begin{array}{c}
\text { LSND } \\
\nu_{\mu} \rightarrow \nu_{e}
\end{array}\right)\left(\begin{array}{l}
\left|\nu_{1}\right\rangle \\
\left|\nu_{2}\right\rangle \\
\left|\nu_{3}\right\rangle \\
\left|\nu_{4}\right\rangle
\end{array}\right),
$$

giving

$$
\left(\begin{array}{l}
\left|\nu_{e}\right\rangle \\
\left|\nu_{s}\right\rangle \\
\left|\nu_{\mu}^{*}\right\rangle \\
\left|\nu_{\tau}^{*}\right\rangle
\end{array}\right)=\left(\begin{array}{cccc}
\cos \phi & \sin \phi \cos \omega & \sin \phi \sin \omega & 0 \\
-\sin \phi & \cos \phi \cos \omega & \cos \phi \sin \omega & 0 \\
0 & -\sin \omega & \cos \omega & 0 \\
0 & 0 & 0 & 1
\end{array}\right)\left(\begin{array}{l}
\left|\nu_{1}\right\rangle \\
\left|\nu_{2}\right\rangle \\
\left|\nu_{3}\right\rangle \\
\left|\nu_{4}\right\rangle
\end{array}\right)
$$

pascos99: submitted to World Scientific on October 30, 2018 
where

$$
\begin{aligned}
& \left|\nu_{\mu}^{*}\right\rangle=1 / \sqrt{2}\left(\left|\nu_{\mu}\right\rangle-\left|\nu_{\tau}\right\rangle\right)=-\sin \omega\left|\nu_{2}\right\rangle+\cos \omega\left|\nu_{3}\right\rangle \\
& \left|\nu_{\tau}^{*}\right\rangle=1 / \sqrt{2}\left(\left|\nu_{\mu}\right\rangle+\left|\nu_{\tau}\right\rangle\right)=\left|\nu_{4}\right\rangle, \text { a mass eigenstate. }
\end{aligned}
$$

In this formalism what occurs at the first level crossing is $\nu_{\mu}^{*} \rightarrow \nu_{s}$, and at the second, $\nu_{e} \rightarrow \nu_{\mu}^{*}$, while $\nu_{\tau}^{*}$ being a mass eigenstate goes through both regions unaffected. Again this gives $1 / 4 \nu_{\mu}, 1 / 4 \nu_{\tau}, 1 / 2 \nu_{s}$, and no $\nu_{e}$ at all.

Note that the $\bar{\nu}_{e}$ flux is also unaffected at the level crossings, so $\bar{\nu}_{e} p \rightarrow e^{+} n$ enhances the neutron number in the $r$ process region, since the protons have not been depleted by $\alpha$ particle formation. It should be emphasized that this mechanism is quite robust, not depending on details of the supernova dynamics, especially as it occurs quite late in the explosive expansion.

It is essential that the two level crossings be in the correct order, and

this provides a requirement on $\Delta m_{e \mu}^{2}$, since the MSW transition depends on density and hence on radial distance from the protoneutron star. Detailed calculations have been made for $\Delta m_{e \mu}^{2} \sim 6 \mathrm{eV}^{2}$, which works very well. Possibly $\Delta m_{e \mu}^{2}$ as low as $2 \mathrm{eV}^{2}$ or maybe even $1 \mathrm{eV}^{2}$ would work, but that is speculative. At any rate, the mass difference needed in this scheme, which is the only one surely consistent with all manifestations of neutrino mass and which rescues the $r$ process, 14 implies appreciable hot dark matter.

\section{Conclusions}

It is quite remarkable that the profound problems of producing the heaviest elements by supernovae can be solved in a manner which requires no adjustment of parameters if the arrangement of masses and mixings of neutrinos is exactly that required to explain the solar $\nu_{e}$ deficit, the atmospheric neutrino anomaly, and the observations of the LSND experiment (or alternatively the need for hot dark matter). This is achieved via an active-sterile level crossing in the supernova, followed by an active-active transition. The total independence of this supernova information strongly enhances the case for this four-neutrino scheme.

\section{Acknowledgments}

This paper is based largely on work done with G.M. Fuller and Y.-Z. Qian, to whom I am grateful, and was supported in part by the U.S. Department of Energy under contract DE-FG03-91ER40618.

pascos99: submitted to World Scientific on October 30, 2018 


\section{References}

1. W.C. Haxton, K. Langanke, Y.-Z. Qian, and P. Vogel, Phys. Rev. Lett. 78, 2694 (1997); Y.-Z. Qian, W.C. Haxton, K. Langanke, and P. Vogel, Phys. Rev. C 55, 1532 (1997); C. Sneden, S. Burles, G.M. Fuller, J.J. Cowan, T. Beers, and D.L. Burris, in preparation (1999).

2. R.D. Hoffman, S.E. Woosley, and Y.-Z. Qian, Astrophys. J. 482, 951 (1996); B.S. Meyer and J.S. Brown, Astrophys. J. Suppl. 112, 199 (1997).

3. G.M. Fuller and B.S. Meyer, Astrophys. J. 453, 792 (1995); B.S. Meyer, G.C. McLaughlin, and G.M. Fuller, Phys. Rev. C 58, 3696 (1998).

4. Y.-Z. Qian and S.E. Woosley, Astrophys. J. 471, 331 (1996); G.M. Fuller and Y.-Z. Qian, Nucl. Phys. A 606, 167 (1996); C.Y. Cardall and G.M. Fuller, Astrophys. J. 486, L111 (1997); J. Samuelson and J.R. Wilson, Astrophys. J., in press (1999); K. Otsuki, H. Tagoshi, T. Kajino, and S. Wanajo, astro-ph/9911164 (to be published in Astrophys. J.).

5. G.C. McLaughlin and G.M. Fuller, Astrophys. J. 472, 440 (1996).

6. D.O. Caldwell, G.M. Fuller and Y.-Z. Qian, astro-ph/9910175 (to be published in Phys. Rev. D).

7. D.O. Caldwell, Perspectives in Neutrinos, Atomic Physics and Gravitation (Editions Frontières, Gif-sur-Yvette, France, 1993), 187.

8. J.R. Primack, J. Holtzman, A. Klypin, and D.O. Caldwell, Phys. Rev. Lett. 74, 2160 (1995); E. Gawiser and J. Silk, Science 280, 1405 (1998).

9. D.O. Caldwell and R.N. Mohapatra, Phys. Rev. D 48, 3259 (1993); J.T. Peltoniemi and J.W.F. Valle, Nucl. Phys. B 406, 409 (1993).

10. C. Athanassopoulos et al, Phys. Rev. Lett. 75, 2650 (1995); Phys. Rev. C 54, 2685 (1996); Phys. Rev. Lett. 77, 3082 (1996); Phys. Rev. Lett. 81, 1774 (1998).

11. K. Eitel, hep-ex/9909036; New Jour. Phys. 2:1.1 (2000).

12. B. Zeitnitz et al., Prog. Part. Nucl. Phys. 40, 169 (1998), but see ref. 11 for more recent information.

13. Y.-Z. Qian et al, Phys. Rev. Lett. 71, 1965 (1993); Y.-Z. Qian and G.M. Fuller, Phys. Rev. D 51, 1479 (1995); G. Sigl, Phys. Rev. D 51, 4035 (1995).

14. G.C. McLaughlin, J.M. Fetter, A.B. Balantekin, and G.M. Fuller, Phys. Rev. C 59, 2873 (1999) employs a neutrino scheme with a heavy sterile neutrino and also rescues the $r$ process, but it is likely to be in trouble with supernova nucleosynthesis, as well as with the Super-Kamiokande atmospheric up-down asymmetry [see S.M. Bilenky, C. Giunti, W. Grimus, and T. Schwatz, Phys. Rev. D 60, 073007 (1999)]. 